This item was submitted to Loughborough's Research Repository by the author.

Items in Figshare are protected by copyright, with all rights reserved, unless otherwise indicated.

\title{
Robust control of nonlinear MAGLEV suspension system with mismatched uncertainties via DOBC approach
}

PLEASE CITE THE PUBLISHED VERSION

http://dx.doi.org/10.1016/j.isatra.2011.01.006

PUBLISHER

Elsevier @ ISA

VERSION

AM (Accepted Manuscript)

LICENCE

CC BY-NC-ND 4.0

REPOSITORY RECORD

Yang, Jun, Argyrios C. Zolotas, Wen-Hua Chen, Konstantinos Michail, and Shihua Li. 2012. "Robust Control of Nonlinear MAGLEV Suspension System with Mismatched Uncertainties via DOBC Approach". figshare. https://hdl.handle.net/2134/11125. 
This item was submitted to Loughborough's Institutional Repository (https://dspace.lboro.ac.uk/) by the author and is made available under the following Creative Commons Licence conditions.

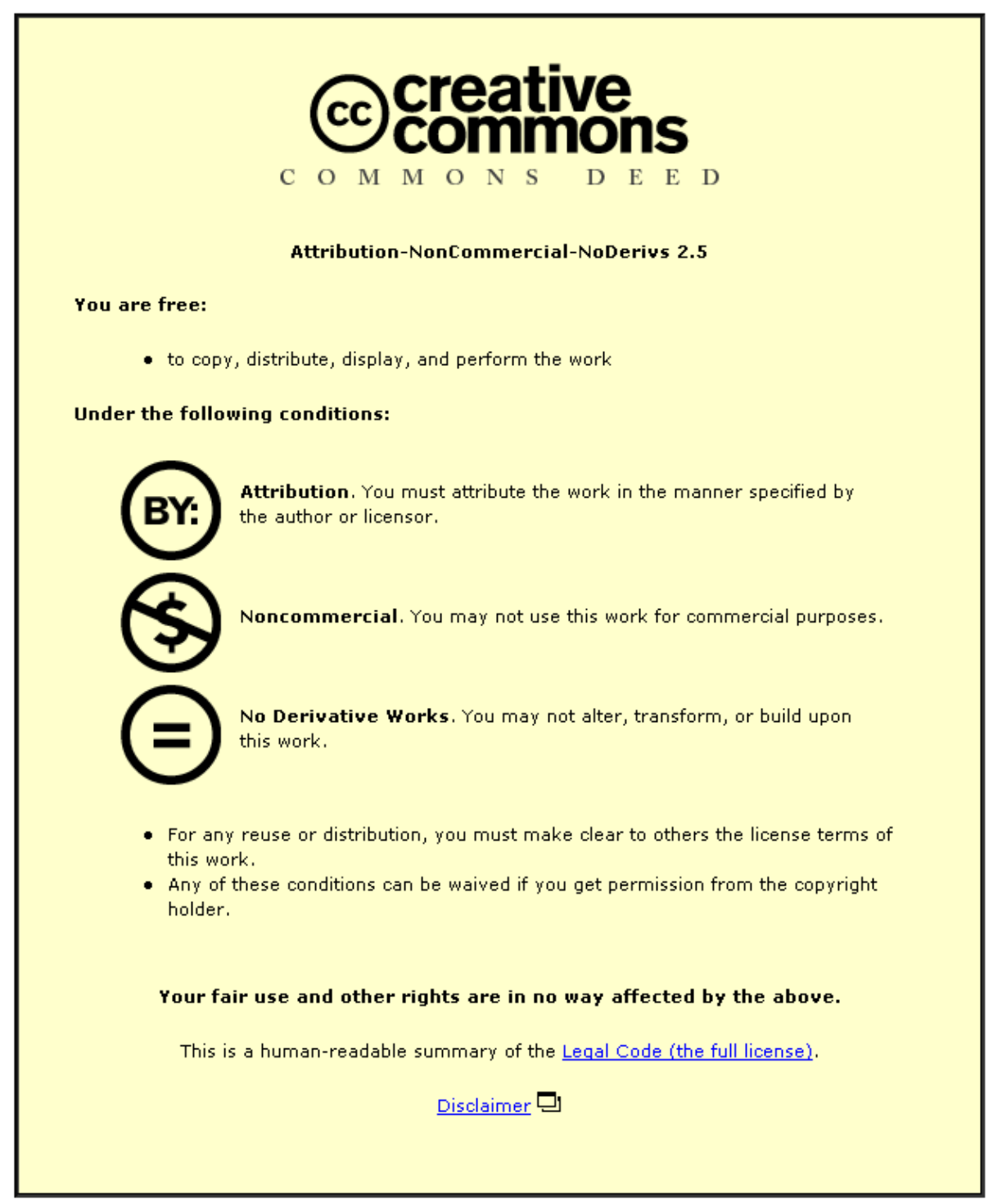

For the full text of this licence, please go to: http://creativecommons.org/licenses/by-nc-nd/2.5/ 


\title{
Robust control of nonlinear MAGLEV suspension system with mismatched uncertainties via DOBC approach
}

\begin{abstract}
Robust control problem of a class of uncertain systems that have disturbances and uncertainties not satisfying "matching" condition, is investigated in this paper via a disturbance observer based control (DOBC) approach. In the context of this paper, "matched" disturbances/uncertainties refers to the disturbances/uncertainties enter the system through the same channels as the control inputs. By properly designing a disturbance compensation gain, a novel composite controller is proposed to counteract the "mismatched" lumped disturbances from the output channels. The proposed method significantly extends the applicability of the DOBC methods. Rigorous stability analysis of the closed-loop system with the proposed method is established under mild assumptions. The proposed method is applied to a nonlinear MAGnetic LEViation (MAGLEV) suspension system. Simulation shows that compared to integral control method, the proposed method provides improved disturbance rejection performance and robustness against load variation.
\end{abstract}

Keywords: "mismatched" disturbances, nonlinear MAGLEV suspension system, state-space disturbance observer, disturbance compensation gain.

\section{Introduction}

Various uncertainties including external disturbances, the effects caused by unmodeled dynamics and parameter variations widely exist in practical systems. These uncertainties often bring adverse effects to closed-loop control systems. With the growing interests in high-precision control, the utilization of disturbance rejection techniques is usually required in control system design. One fact that should be pointed out is many uncertainties in control systems are unmeasurable, thus the disturbance estimation technique could be regarded as highly important for disturbance attenuation.

Disturbance observer is acknowledged as an effective disturbance estimation approach. Disturbance observer based control (DOBC) methods provide powerful abilities in handling system disturbances and improving robustness [1]. The disturbance observer technique was first presented by Ohishi et al. [2] for a position servo system in the late 1980s. During the last three decades, DOBC schemes for linear and nonlinear systems have been proposed and applied successfully in various practical areas, e.g., servo control systems [3, 4], robotic systems [5, 6, 7], hard disk drive systems [8, 9], flight control systems [10, 11], and process control systems [12, 13, 14].

It should be noted that the existing DOBC methods are generally confined to uncertain systems 
which satisfy the so-called "matching" condition $[15,16]$. Here "matching" refers to the uncertainties (also called lumped disturbances) that act on the system via the same channels as the control inputs. The problem of rejecting "mismatched" disturbances (i.e., the disturbances that enter the system through different channels from those of the control inputs) has been looked at in $[17,18]$ where the "matching" disturbances are compensated by DOBC while the "mismatched" disturbances are attenuated by some traditional feedback controllers, such as variable structure control [17] and $H_{\infty}$ control [18]. It is also reported that certain constraints in the "mismatched" disturbances (such as bounded $H_{2}$ norm) are required in $[17,18]$. The most widely used practical method in dealing with "mismatched" disturbances is adding an integral action in a feedback control law to remove offset of the closed-loop system [19], which will be compared with the proposed method in this paper. Note that all above mentioned methods remove the effects of "mismatched" disturbances by feedback regulation in a relatively slow way. It is well known that feedforward control provides satisfactory performance in rejecting disturbances, the significance of which has been shown in the existing DOBC approaches. To this end, it is of great importance to develop a new DOBC method to directly attenuate the "mismatched" disturbances by feedforward compensation rather than by feedback regulation. However, to the best of the authors' knowledge, no systematic DOBC method is presently available to handle the "mismatched" disturbances completely by feedforward compensation.

In addition, it has been noticed that the "mismatched" disturbances are more general and widely exist in practical applications. Taking an aircraft as an example, the lumped disturbance torques caused by unmodeled dynamics, external winds, and parameter perturbations, may affect aircraft dynamics in a rather complicated way, which does not necessarily satisfy the so-called "matching" condition [10]. The problem also appears in a permanent magnet synchronous motor (PMSM) system [20], in which the uncertainties consisting of the parameter variations and the load torque enter the system via different channels to those of the control inputs. Another example is the MAGnetic LEViation (MAGLEV) suspension system, which will be studied in this paper, where the track input disturbances act on different channels from the control input [21, 23].

When confronted with the "mismatched" condition, it is unlikely to counteract the effects of the lumped disturbances completely from the state variables. The design specification in this case is removing the influence of the disturbances from the output channels in steady-state and reducing their influence on transient performance. By properly designing a disturbance compensation gain, a novel DOBC approach is proposed in this paper to solve the disturbance attenuation problem for a class of uncertain systems with "mismatched" disturbances/uncertainties. The proposed method significantly extends the application field of the DOBC approaches. Under the assumption that both the lumped disturbances and their derivatives are bounded, it is shown that the closed-loop system is bounded-input-bounded-output (BIBO) stable with properly designed controller parameters. In addition, rigorous analysis shows that asymptotic stability of the closed-loop system is established under the condition that the lumped disturbances have steady-state values. It is also proved that the 
proposed control method can eliminate the influence of the "mismatched" disturbances/uncertainties of the system from the output channels in steady-state with a properly designed disturbance compensation gain.

During the past few years, MAGLEV suspension system has become one of the most promising transportation systems [24]. Compared with conventional trains, the major superiority of MAGLEV train lies in that the friction, mechanical losses, vibration and noise are reduced substantially since it replaces the wheels by electromagnets and levitates on the guideway and avoids mechanical contact with the rail [25]. However, MAGLEV suspensions are essentially nonlinear systems with lumped disturbances consisting of external disturbances (caused by track input) and parameter perturbations (caused by load variation) [26, 27]. A number of elegant control approaches for MAGLEV systems have been researched throughout the past two decades, including PI control [23], sliding mode control [28], adaptive control [29], robust control [30], H-infinity control [31, 32], and some other traditional methods $[33,21]$. Two reasons motivate the authors to apply the newly proposed DOBC method to the MAGLEV suspension systems. One is most of the feedback based control methods listed above can not react promptly in the presence of strong disturbances since they do not deal with the disturbance directly. The second being the lumped disturbances in MAGLEV systems are "mismatched" ones.

The rest of the paper is organized as follows. The problem of "mismatched" uncertainties in MAGLEV system is illustrated in Section 2. Design and stability analysis of the proposed DOBC method is presented in Section 3. In Section 4, the design of a MAGLEV suspension system using the proposed method is implemented, while concluding remarks are given in Section 5.

\section{Problem formulation}

\subsection{Nonlinear MAGLEV suspension dynamics}

The complete nonlinear model for the MAGLEV suspension system is given by [21, 23],

$$
\begin{gathered}
B=K_{b} \frac{I}{G}, \\
F=K_{f} B^{2} \\
\frac{d I}{d t}=\frac{V_{c}-I R_{c}+\frac{N_{c} A_{p} K_{b}}{G^{2}}\left(\frac{d z_{t}}{d t}-\frac{d Z}{d t}\right)}{\frac{N_{c} A_{p} K_{b}}{G}+L_{c}}, \\
\frac{d^{2} Z}{d^{2} t}=g-\frac{K_{f}}{M_{s}} \frac{I^{2}}{G^{2}} \\
\frac{d G}{d t}=\frac{d z_{t}}{d t}-\frac{d Z}{d t}
\end{gathered}
$$

where variables $I, z_{t}, Z, \frac{d z_{t}}{d t}, \frac{d Z}{d t}, G, B$, and $F$ denote the current, the rail position, the electromagnet position, the rail vertical velocity, the electromagnet vertical velocity, the air gap, the flux density, and the force, respectively. Signal $V_{c}$ is the voltage of the coil. Remaining symbols in Eqs. (1)-(5) are system parameters which are listed in Table 1. 
Table 1: Parameters of MAGLEV suspension system

\begin{tabular}{|l|l|l|}
\hline Parameters & Meaning & Value \\
\hline$M_{s}$ & Carriage Mass & $1000 \mathrm{~kg}$ \\
$K_{b}$ & Flux coefficient & $0.0015 \mathrm{~T} \cdot \mathrm{m} / \mathrm{A}$ \\
$K_{f}$ & Force coefficient & $9810 \mathrm{~N} / \mathrm{T}^{2}$ \\
$R_{c}$ & Coil's Resistance & $10 \Omega$ \\
$g$ & Gravity constant & $9.81 \mathrm{~m} / \mathrm{s}^{2}$ \\
$L_{c}$ & Coil's Inductance & $0.1 \mathrm{H}$ \\
$N_{c}$ & Number of turns & 2000 \\
$A_{p}$ & Pole face area & $0.01 \mathrm{~m}^{2}$ \\
\hline
\end{tabular}

\subsection{Model linearization}

The linearization of the MAGLEV suspension is based on small perturbations around the operating point $[23,27]$. The following definitions are used in which the lower case letters define a small variation around the operating point and the subscript 'o' refers to the operating condition.

$$
\begin{gathered}
B=B_{o}+b, \\
F=F_{o}+f, \\
I=I_{o}+i, \\
G=G_{o}+\left(z_{t}-z\right), \\
V_{c}=V_{o}+u_{c},
\end{gathered}
$$

Table 2: Nominal values of MAGLEV suspension system

\begin{tabular}{|l|l|l|}
\hline Parameters & Meaning & Value \\
\hline$B_{O}$ & Nominal flux density & $1.0 \mathrm{~T}$ \\
$F_{o}$ & Nominal force & $9810 \mathrm{~N}$ \\
$I_{O}$ & Nominal current & $10 \mathrm{~A}$ \\
$G_{O}$ & Nominal air gap & $0.015 \mathrm{~m}$ \\
$V_{O}$ & Nominal voltage & $100 \mathrm{~V}$ \\
\hline
\end{tabular}

The nominal values of the variables in operating point are given in Table 2. The nonlinear MAGLEV suspension models (3)-(5) are expressed as a linear system with disturbances and uncertainties

$$
\left\{\begin{array}{l}
\dot{x}=A x+B_{u} u+B_{d} d+\Delta A x+O(x, u, d), \\
y=C x
\end{array}\right.
$$


where the states are the linearized current, vertical carriage velocity and air gap, i.e., $x=\left[i \dot{z}\left(z_{t}-\right.\right.$ $z)]^{T}$, the input $u=u_{c}$ is the voltage, the track input disturbance $d=\dot{z}_{t}$ is the rail vertical velocity (the external disturbance). The controlled variable is the variation of the air gap, i.e., $y=z_{t}-z$. $\Delta A$ is the perturbation matrix caused by load variation. Nonlinear function $O(x, u, d)$ consists of the high order nonlinear terms with respect to $x, u$ and $d$. The detailed liberalization procedure can be found in [23]. The matrixes $A, B_{u}, B_{d}$ and $C$ are given as

$$
\begin{aligned}
& A=\left[\begin{array}{ccc}
\frac{-R_{c}}{L_{c}+K_{b} N_{c} \frac{A_{p}}{G_{o}}} & \frac{-K_{b} N_{c} A_{p} I_{o}}{G_{o}^{2}\left(L_{c}+K_{b} N_{c} \frac{A_{p}}{G_{o}}\right)} & 0 \\
-2 K_{f} \frac{I_{o}}{M_{s} G_{o}^{2}} & 0 & 2 K_{f} \frac{I_{o}^{2}}{M_{s} G_{o}^{3}} \\
0 & -1 & 0
\end{array}\right], \\
& B_{u}=\left[\begin{array}{c}
\frac{1}{L_{c}+K_{b} N_{c} \frac{A_{p}}{G_{o}}} \\
0 \\
0
\end{array}\right] \\
& B_{d}=\left[\begin{array}{c}
\frac{K_{b} N_{c} A_{p} I_{o}}{G_{o}^{2}\left(L_{c}+K_{b} N_{c} \frac{A_{p}}{G_{o}}\right)} \\
0 \\
1
\end{array}\right] \text {, } \\
& C=\left[\begin{array}{lll}
0 & 0 & 1
\end{array}\right] .
\end{aligned}
$$

Remark 1. In (11), the high order nonlinear function $O(x, u, d)$ is generally neglected when designing controllers since their magnitudes are much smaller as compared with the linear parts. In this paper, such high order nonlinear terms $O(x, u, d)$ are no longer neglected but considered as part of the lumped disturbances.

The control specifications of the MAGLEV suspension under consideration of deterministic track input are given in Table 3.

Table 3: Control specifications for MAGLEV suspension system (deterministic)

\begin{tabular}{|l||l|}
\hline Constraints & Value \\
\hline Maximum air gap deviation, $\left(z_{t}-z\right)_{p}$ & $\leq 0.0075 \mathrm{~m}$ \\
Maximum input coil voltage, $\left(u_{c}\right)_{p}$ & $\leq 300 \mathrm{~V}\left(3 I_{o} R_{c}\right)$ \\
Settling time, $t_{s}$ & $\leq 3 \mathrm{~s}$ \\
Air gap steady state error, $\left(z_{t}-z\right)_{e_{s s}}$ & $=0$ \\
\hline
\end{tabular}

\section{3 "Mismatched" disturbances/uncertainties}

In MAGLEV suspension systems, the lumped disturbances (or uncertainties) are formulated by merging the effects caused by parameter variation and nonlinearities into the disturbance terms, 
which are described as

$$
d_{l}=B_{d} d+\Delta A x+O(x, u, d) .
$$

Substituting (16) into (11), the full dynamic model of the nonlinear MAGLEV suspension system is described as

$$
\left\{\begin{array}{l}
\dot{x}=A x+B_{u} u+B_{l d} d_{l}, \\
y=C x,
\end{array}\right.
$$

where $B_{l d}=I$ is a $3 \times 3$ identity matrix.

Remark 2. Note that $d_{l}$ in (17), which is a vector with the dimension three, includes system uncertainties consisting of the external disturbance and the effects caused by parameter variation and nonlinearities.

Remark 3. It can be observed from Eqs. (13) and (17) that there are multiple disturbances but a single control input. Hence, the disturbances definitely enter the system via different channel from the control input, forming a set of "mismatched" disturbances.

Besides in MAGLEV systems, the "mismatched" disturbances/uncertainties also exist in other practical industrial control systems, e.g., missile system [10] and motor system [20]. In the presence of "mismatched" disturbances/uncertainties, the existing DOBC methods are no longer available. This is better explained via the following example. Considering a simple system, i.e.,

$$
\left\{\begin{array}{l}
\dot{x}_{1}=x_{2}+d, \\
\dot{x}_{2}=x_{1}+x_{2}+u, \\
y=x_{1} .
\end{array}\right.
$$

For system (18), the estimate $\hat{d}$ of the real disturbance $d$ can be obtained by a disturbance observer. However, if the composite control law is designed as $u=K_{x} x-\hat{d}$ (where $K_{x}$ is the feedback control gain) which is the formulation of the existing DOBC methods from the literature, it can be found that the disturbance compensation design has nothing meaningful for system (18) because the disturbance can neither be attenuated from the state equations nor from the output channels.

It should be pointed out that the "mismatched" disturbances are unlikely to be attenuated from the state equations in general. This work aims to develop a disturbance compensation gain so that the DOBC method can be used to remove "mismatched" disturbances/uncertainties from the output channels.

\section{A novel DOBC for "mismatched" uncertainties}

Consider a multi-input-multi-output (MIMO) linear system with multiple disturbances, depicted by

$$
\left\{\begin{array}{l}
\dot{x}=A x+B_{u} u+B_{l d} d_{l}, \\
y=C x,
\end{array}\right.
$$

where $x \in R^{n}, u \in R^{m}, d_{l} \in R^{r}$, and $y \in R^{s}$ are state, control input, lumped disturbance, and output vectors, respectively. The system matrixes $A, B_{u}, B_{l d}$, and $C$ are with dimensions of $n \times n$, $n \times m, n \times r$, and $s \times n$, respectively. 
Remark 4. In (19), the lumped disturbances are generalised concepts of disturbances, possibly including external disturbances, unmolded high order dynamics, parameter variations, and nonlinear dynamics that can not be captured by the linear dynamics in Eq. (19).

For system (19), the following state-space disturbance observer $[5,7,10,22]$ is designed to estimate the disturbances

$$
\left\{\begin{array}{l}
\dot{p}=-L B_{l d}(p+L x)-L\left(A x+B_{u} u\right), \\
\hat{d}_{l}=p+L x
\end{array}\right.
$$

where $\hat{d}_{l}$ is the disturbance estimate vector, $p$ is an auxiliary vector and $L$ is the observer gain matrix to be designed.

Remark 5. The state-space DOB presented in (20) can be used to estimate both "matched" and "mismatched" uncertainties.

Based on the disturbance estimate by DOB, a new composite control law is proposed, i.e.,

$$
u=K_{x} x+K_{d} \hat{d}_{l},
$$

An appropriately designed disturbance compensation gain $K_{d}$ guarantees that the influence of the disturbances can be removed from the output channels in the steady-state.

For system (19) subject to "mismatched" uncertainties, a general design procedure of the proposed DOBC method is given below:

1) Design a feedback controller to achieve stability without considering the lumped disturbances.

2) Design a linear state-space disturbance observer to estimate the lumped disturbances.

3) Design a disturbance compensation gain to guarantee that the "mismatched" lumped disturbances are removed from the output channels in steady-state.

4) Integrate the feedback controller and the feedforward compensator to formulate the composite DOBC law.

To establish the stability of the closed-loop system, some mild assumptions are given as follows.

Assumption 1. Both the lumped disturbance $d_{l}$ and its derivative $\dot{d}_{l}$ are bounded.

Assumption 2. The lumped disturbances $d_{l}$ have constant values in steady-state, i.e., $\lim _{t \rightarrow \infty} \dot{d}_{l}(t)=0$ or $\lim _{t \rightarrow \infty} d_{l}(t)=d_{s}$ where $d_{s}$ is a constant vector.

Assumption 3. $\left(A, B_{u}\right)$ is controllable.

The asymptotic stability of DOB (20) is concluded by the following theorem.

Theorem 1. Suppose that Assumptions 1 and 2 are satisfied for system (19). The disturbance estimates $\hat{d}_{l}$ yielded by DOB (20) can asymptotically track the lumped disturbances $d_{l}$ if the observer gain matrix $L$ in (20) is chosen such that matrix $-L B_{l d}$ is Hurwitz.

Proof. The disturbance estimation error of the DOB (20) is defined as

$$
e_{d}=\hat{d}_{l}-d_{l}
$$


Combining system (19), DOB (20), with estimation error (22), gives

$$
\begin{aligned}
\dot{e}_{d} & =\dot{\hat{d}}_{l}-\dot{d}_{l} \\
& =\dot{p}+L \dot{x}-\dot{d}_{l} \\
& =-L B_{l d} \hat{d}_{l}-L\left(A x+B_{u} u\right)+L\left(A x+B_{u} u+B_{l d} d_{l}\right)-\dot{d}_{l} \\
& =-L B_{l d}\left(\hat{d}_{l}-d_{l}\right)-\dot{d}_{l} \\
& =-L B_{l d} e_{d}-\dot{d}_{l} .
\end{aligned}
$$

It can be verified that the error system (23) is asymptotically stable since $-L B_{l d}$ is Hurwitz, $\dot{d}_{l}$ is bounded and satisfies $\lim _{t \rightarrow \infty} \dot{d}_{l}=0$. This implies that the disturbance estimate of DOB can track the disturbances asymptotically.

Remark 6. It seems that the asymptotic tracking of DOB can only obtained based on the assumption that $\lim _{t \rightarrow \infty} \dot{d}_{l}(t)=0$. However, it is pointed out in [5] that the estimates by DOB (20) can also track some fast time-varying disturbances well as long as the observer dynamics are much faster than those of the disturbances.

Remark 7. In the presence of uncertainties, the lumped disturbances would be a function of the states, which can be reasonably estimated if the DOB dynamics are faster than the closed-loop dynamics. The same argument for the state observer based control methods is applicable.

The bounded-input-bounded-output (BIBO) and asymptotic stabilities of the closed-loop system are shown as follows.

Theorem 2. Suppose that Assumptions 1 and 3 are satisfied for system (19). The BIBO stability of system (19) under the newly proposed DOBC law (21) is guaranteed if the observer gain $L$ in (20) and the feedback control gain $K_{x}$ in (21) are selected such that both $-L B_{l d}$ and $A+B_{u} K_{x}$ are Hurwitz.

Proof. Combining system (19), composite control law (21), with error system (23), the closedloop system is written as

$$
\left[\begin{array}{c}
\dot{x} \\
\dot{e}_{d}
\end{array}\right]=\left[\begin{array}{cc}
A+B_{u} K_{x} & B_{u} K_{d} \\
0 & -L B_{l d}
\end{array}\right]\left[\begin{array}{c}
x \\
e_{d}
\end{array}\right]+\left[\begin{array}{cc}
B_{u} K_{d}+B_{l d} & 0 \\
0 & -1
\end{array}\right]\left[\begin{array}{c}
d_{l} \\
\dot{d}_{l}
\end{array}\right] .
$$

Since $-L B_{l d}$ and $A+B_{u} K_{x}$ are Hurwitz, it can be verified that matrix

$$
\left[\begin{array}{cc}
A+B_{u} K_{x} & B_{u} K_{d} \\
0 & -L B_{l d}
\end{array}\right]
$$

is also Hurwitz. It can be concluded that the closed-loop system (24) is BIBO stable for any bounded $d_{l}$ and $\dot{d}_{l}$ if $K_{x}$ and $L$ are properly selected.

Theorem 3. Suppose that Assumptions 1 and 2 are satisfied for system (19). The state of system (19) under the composite control law (21) converges to $x_{s}=-\left(A+B_{u} K_{x}\right)^{-1}\left(B_{u} K_{d}+B_{l d}\right) d_{s}$ asymptotically if the observer gain $L$ in (20) and the feedback control gain $K_{x}$ in (21) are selected such that both $-L B_{l d}$ and $A+B_{u} K_{x}$ are Hurwitz. 
Proof. The state error can be constructed as

$$
e_{x}=x-x_{s}
$$

Combining system (19), composite control law (21), error system (23), with state error (25), the closed-loop system are given as

$$
\left[\begin{array}{c}
\dot{e}_{x} \\
\dot{e}_{d}
\end{array}\right]=\left[\begin{array}{cc}
A+B_{u} K_{x} & B_{u} K_{d} \\
0 & -L B_{l d}
\end{array}\right]\left[\begin{array}{c}
e_{x} \\
e_{d}
\end{array}\right]+\left[\begin{array}{cc}
B_{u} K_{d}+B_{l d} & 0 \\
0 & -1
\end{array}\right]\left[\begin{array}{c}
d_{l}-d_{s} \\
\dot{d}_{l}
\end{array}\right] .
$$

Similar with the proof of Theorem 2, it can be verified that

$$
\left[\begin{array}{cc}
A+B_{u} K_{x} & B_{u} K_{d} \\
0 & -L B_{l d}
\end{array}\right]
$$

is Hurwitz. In addition, it can be obtained from Assumption 2 that $\lim _{t \rightarrow \infty}\left[d_{l}(t)-d_{s}\right]=0$.

Considering the conditions, it can be shown that the closed-loop system (26) is asymptotically stable. This means that with the given conditions the state vector converges to a constant vector $x_{s}$ asymptotically.

The main theoretical contribution of this work is that a systematic method is developed for disturbance compensation gain design so that the disturbances can be attenuated from the output channels in steady-state.

Assumption 4. The system matrixes and the feedback control gain satisfy the rank condition that $\operatorname{rank}\left(C\left(A+B_{u} K_{x}\right)^{-1} B_{u}\right)=\operatorname{rank}\left(\left[C\left(A+B_{u} K_{x}\right)^{-1} B_{u},-C\left(A+B_{u} K_{x}\right)^{-1} B_{l d}\right]\right)$.

Theorem 4. Suppose that Assumptions 1-4 are satisfied for system (19), also the observer gain $L$ and the feedback control gain $K_{x}$ are chosen to make matrixes $-L B_{l d}$ and $A+B_{u} K_{x}$ Hurwitz. Considering system (19) under the proposed composite control law (21), the lumped disturbances can be attenuated from the output channel in steady-state if the disturbance compensation gain is designed such that

$$
C\left(A+B_{u} K_{x}\right)^{-1} B_{u} K_{d}=-C\left(A+B_{u} K_{x}\right)^{-1} B_{l d}
$$

Proof. Substituting control law (21) into system (19), the state is expressed as

$$
x=\left(A+B_{u} K_{x}\right)^{-1}\left[\dot{x}-B_{u} K_{d} \hat{d}_{l}-B_{l d} d_{l}\right] .
$$

Combining (19), (27) with (28), gives

$$
y=C\left(A+B_{u} K_{x}\right)^{-1} \dot{x}+C\left(A+B_{u} K_{x}\right)^{-1} B_{l d}\left(\hat{d}_{l}-d_{l}\right)
$$

With the given conditions, the following results are obtained according to Theorem 3 ,

$$
\lim _{t \rightarrow \infty} e_{x}(t)=0, \lim _{t \rightarrow \infty} e_{d}(t)=0 .
$$


Under the consideration of (30), taking limits of both sides of Eq. (29) with respect to $t$, gives

$$
\lim _{t \rightarrow \infty} y(t)=0 .
$$

Remark 8. The disturbance compensation gain $K_{d}$ in (27) is a general case and suitable for both "matching" and "mismatched" disturbances. In the "matching" case, i.e., $B_{u}=B_{l d}$, it can be obtained from (27) that the disturbance compensation gain is reduced to $K_{d}=-1$ which is the particular form of most existing DOBC methods.

\section{Application to MAGLEV suspension system}

\subsection{Controller design}

It should be pointed out that any feedback controller that can stabilize system (17) in the absence of disturbances may be used to achieve tracking performance. In the context of this paper, we concentrate on deterministic design only. In this study, the classical linear quadratic regulator (LQR) is employed and the penalty matrixes $Q$ and $R$ in the cost function of LQR are simply selected as

$$
Q=\left[\begin{array}{lll}
1 & 0 & 0 \\
0 & 1 & 0 \\
0 & 0 & 1
\end{array}\right], R=0.1 .
$$

To this end, the feedback control gain of LQR can be calculated according to the model information and the penalty matrixes, given as $K_{x}=[-61-59140061]$. With the parameter matrixes given in (12), (13), (17) and the calculated control gain $K_{x}$, the disturbance compensation gain is calculated by Eq. (27), given as $K_{d}=[-2.136 .0742 .2]$.

To guarantee the asymptotic stability of the DOB, the observer gain matrix in (20) is designed as

$$
L=\left[\begin{array}{ccc}
40 & 0 & 0 \\
0 & 40 & 0 \\
0 & 0 & 40
\end{array}\right] .
$$

The control structure of the proposed DOBC method for the nonlinear MAGLEV suspension system is shown in Fig. 1.

\subsection{Simulations and analysis}

In the presence of disturbances/uncertainties, many control methods (e.g. LQR) can not remove the offset. The integral control is a widely used practical method to eliminate the steady-state error in these cases. To demonstrate the effectiveness of the proposed method, LQR plus an integral action (called LQR+I) method is employed for comparison. The control law of the LQR+I method is represented as

$$
u_{l q r+i}(t)=K_{x} x(t)+k_{i} \int_{t_{0}}^{t}\left[z_{t}(\tau)-z(\tau)\right] d \tau,
$$




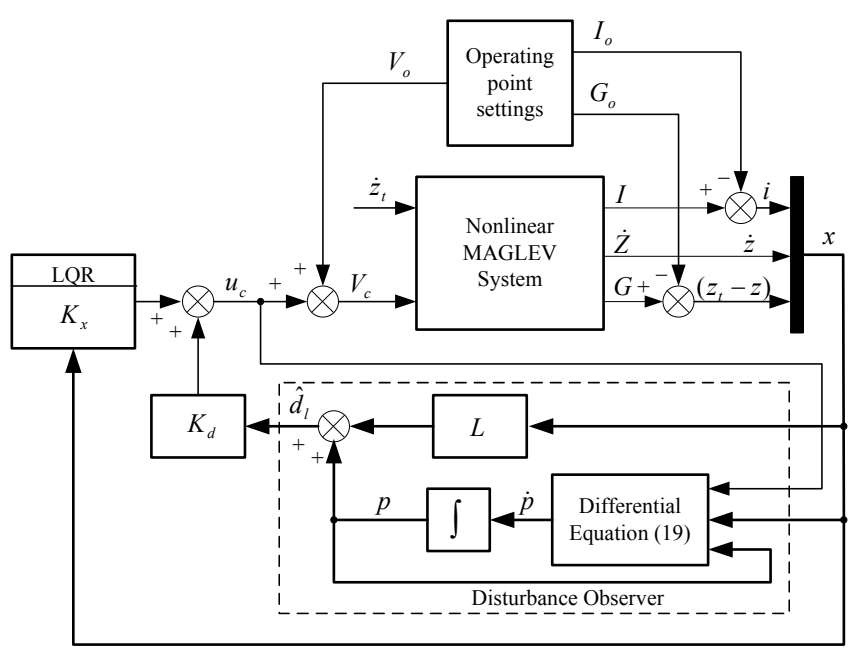

Figure 1: Block diagram of the proposed DOBC method for the nonlinear MAGLEV system.

where $t_{0}$ is the initial time and $k_{i}$ is the integral coefficient to be designed. To find an appropriate integral coefficient, a variable $x_{4}=\int_{t_{0}}^{t}\left[z_{t}(\tau)-z(\tau)\right] d \tau$ is taken as an augmented state. For the augmented system, the LQR method is used to get the control gain. The penalty matrixes are selected as

$$
Q_{I}=\left[\begin{array}{cc}
Q & 0 \\
0 & q_{i}
\end{array}\right], R_{I}=R=0.1
$$

Set $q_{i}=1.0 \times 10^{9}$, then the integral coefficient is able to be calculated and gives as $k_{i}=-1.0 \times 10^{5}$.

\subsubsection{External disturbance rejection performance}

The main external disturbances in the MAGLEV system are the deterministic inputs to the suspension in the vertical direction. Such deterministic inputs are the transitions onto track gradients. In this paper, the deterministic input components considered are referred to [23] and shown in Fig. 2. They represent a gradient of $5 \%$ at a vehicle speed of $15 \mathrm{~m} / \mathrm{s}$ and an allowed acceleration of 0.5 $\mathrm{m} / \mathrm{s}^{2}$ while the jerk level is $1 \mathrm{~m} / \mathrm{s}^{3}$.

The response curves of both the output and input of the suspension system under the proposed DOBC method (solid line), LQR+I method (dashed line) and LQR method (dash-dotted line) are shown in Fig. 3. Response curves of the corresponding states are shown in Fig. 4. Note that zero air gap variation refers to $G_{o}$, as we follow the treatment setup proposed in $[21,26,27]$.

As shown in Figs. 3 and 4, the LQR method results in unstable control of the airgap and current in such case of external disturbances. It can be observed from Fig. 3(a) that both the overshoot and settling time under the DOBC method are shorter than those under the LQR+I method, the convergence rate under the DOBC method is much faster than that under the LQR+I method. As shown in Fig. 3(b), the maximum input voltage under the DOBC method is smaller than that under the LQR+I method. Response curves in Fig. 4 show that both the current and the vertical electromagnet velocity under the DOBC method vary smoothly and approach to the desired equilibrium points faster than 


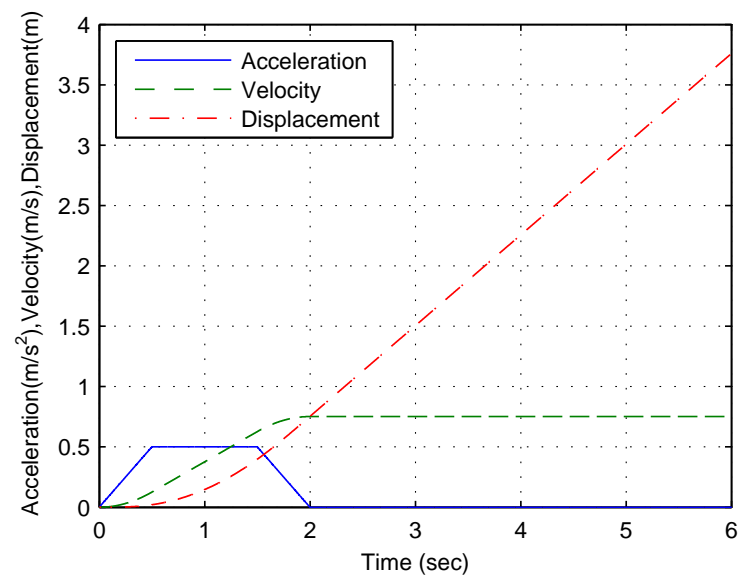

Figure 2: Track (deterministic) input to the suspension with a vehicle speed of $15 \mathrm{~m} / \mathrm{s}$ and $5 \%$ gradient.

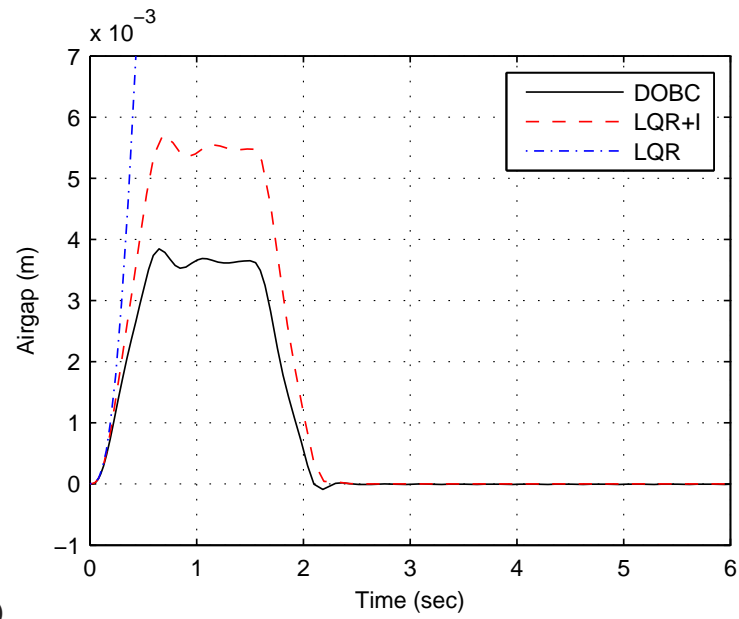

(a)

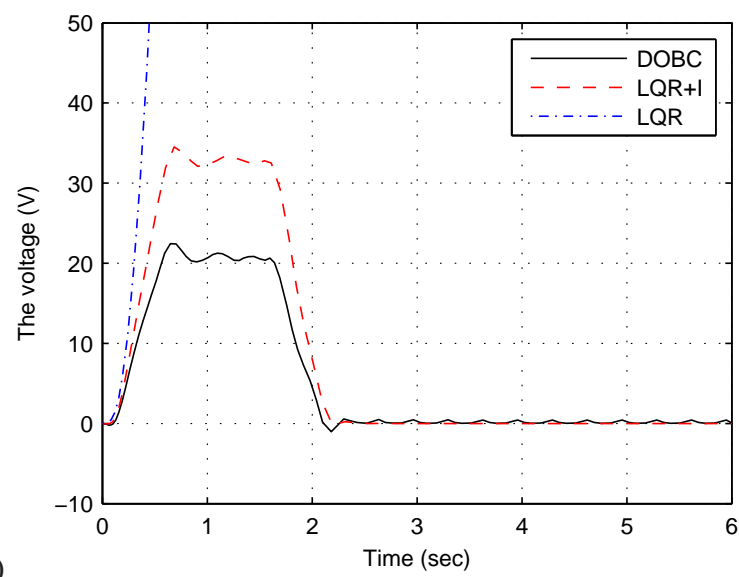

Figure 3: Response curves of the input and output in the presence of deterministic track input: (a) the air gap, $\left(z_{t}-z\right)$, (b) the voltage of the coil , $u_{c}$. 


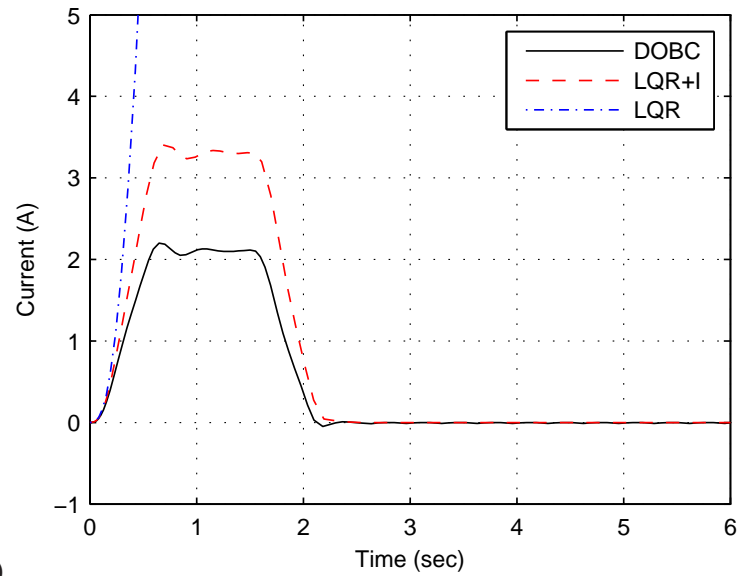

(a)

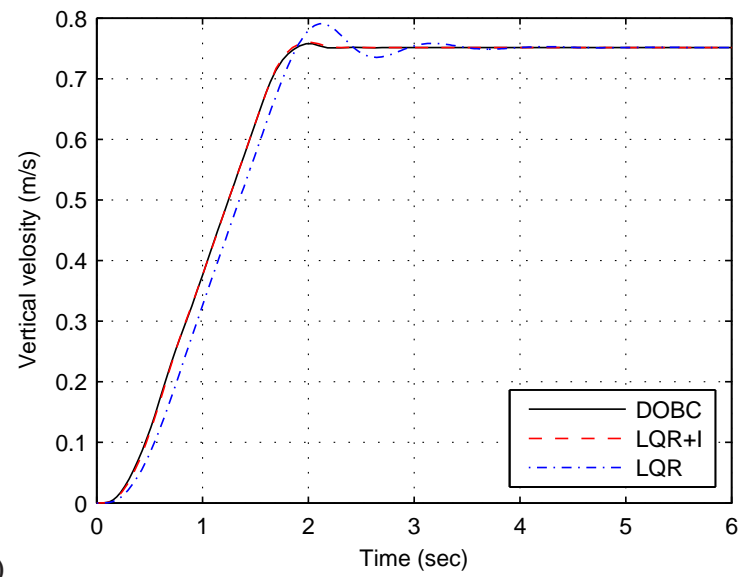

(b)

Figure 4: Response curves of the states in the presence of deterministic track input: (a) the current, $i$, (b) the vertical electromagnet velocity, $\dot{z}$. 
that under the LQR+I method. The results demonstrate that the newly proposed DOBC method has achieved much better performance in rejecting such practical disturbances than that of the traditional LQR+I method.

\subsubsection{Robustness against load variation}

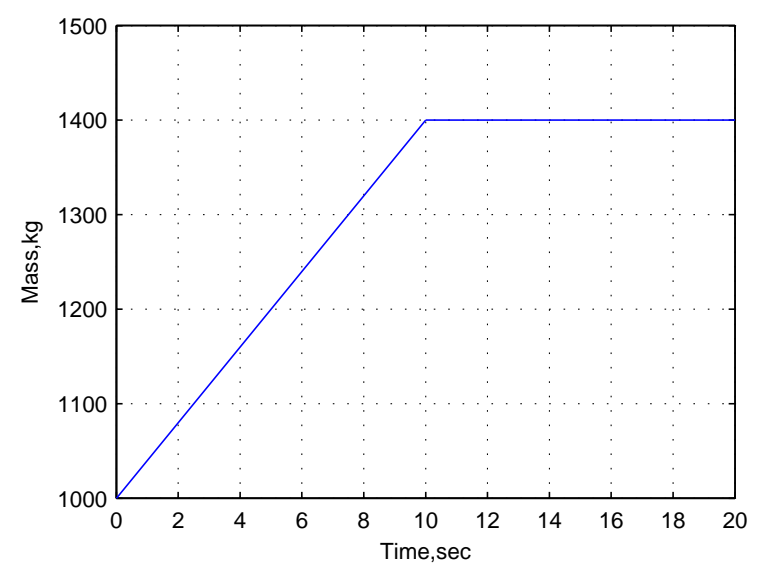

Figure 5: Curve of the load variation.

In this part, the load variation of the MAGLEV suspension is considered. The suspension has to support the large mass of the vehicle as well as the load (weight of the passengers) which can vary up to $40 \%$ of the total mass of the vehicle [23]. This is a considerable variation of the total mass and may results in undesirable performance. To this end, the robustness against load variations should be taken into account to ensure performance and stability for a fully laden or unladen vehicle. For testing, it is supposed that the load variation is up to $40 \%$ of the total vehicle mass within 10 seconds, i.e., the load can vary from $1000 \mathrm{~kg}$ to $1400 \mathrm{~kg}$ for a fully unladen and laden vehicle, respectively. The profile of the load variation is shown in Fig. 5. The track input disturbance like in Fig. 2 is also considered but acts on the system at $t=15 \mathrm{~s}$.

The robustness against such case of load variation under both DOBC (solid line), LQR+I (dashed line) and LQR (dash-dotted line) methods can be seen in Figs. 6 and 7. It can be found that the LQR method leads to unstable control of the closed-loop system in such cases of uncertainties. It can be observed from Fig. 6(a) that the overshoot of the air gap deviation under DOBC method is much smaller than that under the LQR+I method. In addition, the convergence rate under the DOBC method is much faster than that under the LQR+I method. Fig. 6(b) shows that the magnitude of the coil voltage under DOBC method is smaller than that under the LQR+I method. Test results in this subsection manifest that the proposed method obtains promising performance of robustness against load variation as compared with that of the integral control method. 


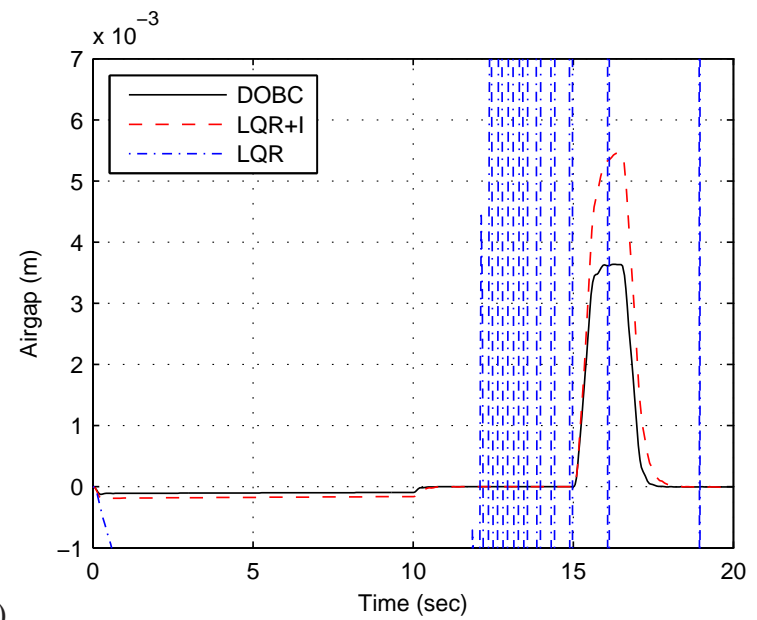

(a)

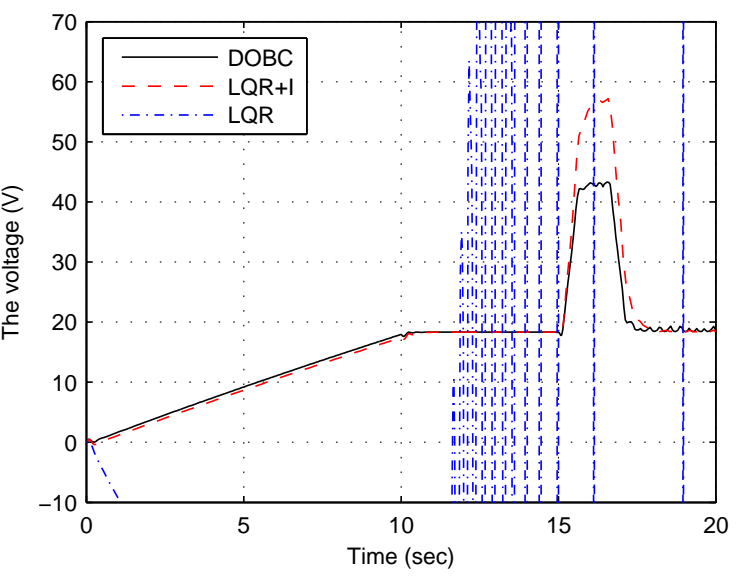

Figure 6: Response curves of the input and output in the presence of load variation: (a) the air gap, $\left(z_{t}-z\right)$, (b) the voltage of the coil, $u_{c}$. 


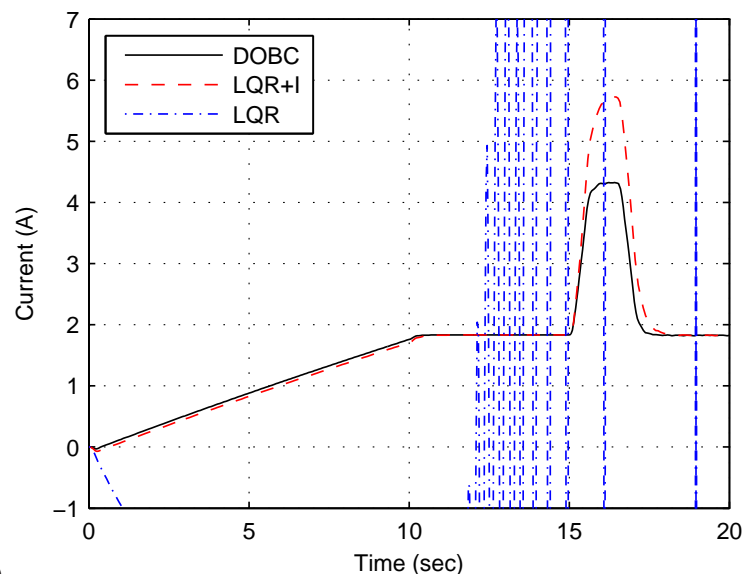

(a)

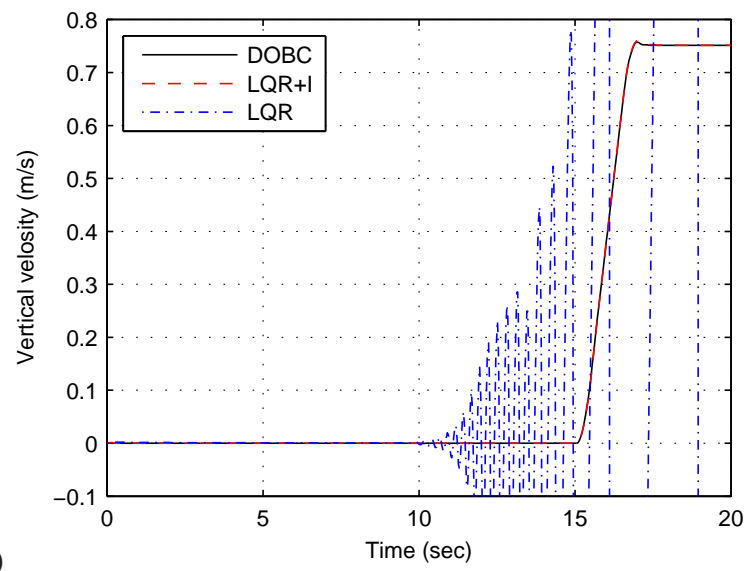

Figure 7: Response curves of the states in the presence of load variation: (a) the current, $i$, (b) the vertical electromagnet velocity, $\dot{z}$. 


\section{Concluding remarks}

By appropriately designing a disturbance compensation gain, a novel disturbance observer based control (DOBC) method has been proposed to solve the disturbance rejection problem of the uncertain system which contains "mismatched" disturbances. Here "mismatched" means the lumped disturbances enter the system through different channels from the control inputs. With the proposed control method, rigorous stability of the closed-loop system has been analyzed under some soundable assumptions. It has also been verified that the "mismatched" lumped disturbances can be attenuated from the output channels with the properly designed disturbance compensation gain. To shown the feasibility and efficiency of the proposed method, application design is carried out for an industrial MAGLEV suspension system, which is essentially a system with "mismatched" disturbances including external disturbances (caused by track inputs), nonlinear dynamics (neglected nonlinearities during liberalization) and parameter perturbations (caused by load variation). When control such practical MAGLEV suspension system, simulation has shown that the proposed method achieves much better performance of external disturbance rejection and robustness against load variation than those of the traditional integral control method.

\section{References}

[1] Shim H, Jo NH. An almost necessary and sufficient condition for robust stability of closed-loop systems with disturbance observer. Automatica 2009; 45(1): 296-299.

[2] Ohishi K, Nakao M, Ohnishi K, Miyachi K. Microprocessor-controlled DC motor for loadinsensitive position servo system. IEEE Trans Ind Electron 1987; 31(1): 44-49.

[3] Yao B, Majed MA, Tomizuka M. High-Performance robust motion control of machine tools: an adaptive robust control approach and comparative experiments. IEEE/ASME Trans Mechatron 1997; 2(2): 63-76.

[4] Kempf CJ, Kobayashi S. Disturbance observer and feedforward design for a high-speed directdrive position table. IEEE Trans Control Syst Technol 1999; 7(5): 513-526.

[5] Chen W-H, Gawthrop PJ, Reilly JO. A nonlinear disturbance observer for robotic manipulators. IEEE Trans Ind Electron 2000; 47(4): 932-938.

[6] Komada S, Machii N, Hori T. Control of redundant manipulators considering order of disturbance observer. IEEE Trans Ind Electron 2000:47(2): 413-420.

[7] Chen W-H. Disturbance observer based control for nonlinear systems. IEEE/ASME Trans Mechatron 2004; 9(4): 706-710.

[8] Ishikawa J, and Tomizuka M. A novel add-on compensator for cancellation of pivot nonlinearities in hard disk drives. IEEE Trans Magn 1998; 34(4): 1895-1897.

[9] Thum CK, Du C, Lewis FL, Chen BM, Ong EH. H-infinity disturbance observer design for high precision track following in hard disk drives. IET Contr Theory Appl 2009; 3(12): 15911598.

[10] Chen W-H. Nonlinear disturbance observer-enhanced dynamic inversion control of missiles. J Guid Control Dyn 2003; 26(1): 161-166.

[11] Guo L, Chen W-H. Disturbance attenuation and rejection for systems with nonlinearity via DOBC approach. Int J Robust Nonlinear Control 2005; 15(3): 109-125.

[12] Chen XS, Yang J, Li SH, Li Q. Disturbance observer based multi-variable control of ball mill grinding circuits. J Process Control 2009; 19(7): 1205-1213.

[13] Matausek MR, Ribic AI. Design and robust tuning of control scheme based on the PD controller plus disturbance observer and low-order integrating first-order plus dead-time model. ISA T 2009; 48(4): 410-416.

[14] Yang J, Li SH, Chen XS, Li Q. Disturbance rejection of ball mill grinding circuits using DOB and MPC. Powder Technol 2010; 198(2): 219-228. 
[15] Barmish BR, Leitmann G. On ultimate boundness control of uncertain systems in the absence of matching assumptions. IEEE Trans Autom Control 1982; 27(1): 153-158.

[16] Gutman S, Letmann G. Stabilizing feedback control for dynamical systems with bounded uncertainties. In Proc. IEEE Conf. Decision and Control. 1976. p.94-99.

[17] Wei XJ, Guo L. Composite disturbance-observer-based control and terminal sliding model control for non-linear systems with disturbances. Int J Control 2009; 82(6): 1082-1098.

[18] Wei XJ, Guo L. Composite disturbance-observer-based control and H-infinity control for complex continuous models. Int J Robust Nonlinear Control 2010; 20(1): 106-118.

[19] Lin C-F, Cloutier JR, Evers JH. High-performance, robust, bank-to-turn missile autopilot design. J Guid Control Dyn 1995; 18(1): 46-53.

[20] Mohamed YA-RI. Design and implementation of a robust current-control scheme for a PMSM vector drive with a simple adaptive disturbance observer. IEEE Trans Ind Electron 2007; 54(4): 1981-1988.

[21] MacLeod C, Goodall RM. Frequency-shaping LQ control of Maglev suspension systems for optimal performance with deterministic and stochastic inputs. IEE Proc-Control Theory Appl 1996; 143(1): 25-30.

[22] Chen W-H, Ballance DJ, Gawthrop PJ, Gribble JJ, O’Reilly J. Nonlinear PID predictive controller. IEE Proc-Control Theory Appl 1999; 146(6): 603-611.

[23] Michail K. Optimised Configuration of Sensing Elements for Control and Fault Tolerance Applied to An Elctro-Magnetic Suspension System. PhD thesis, Loughborough University, 2009 (http://hdl.handle.net/2134/5806)

[24] Lee H-W, Kim K-C, Lee J. Review of Maglev train technology. IEEE Trans Magn 2006; 42(7): 1917-1925.

[25] Michail K, Zolotas A, Goodall R. Optimised sensor configurations for a Maglev suspension system. IFAC Proc Vol 2008; 17(1): 169-184.

[26] Goodall R. Dynamics and control requirements for EMS Maglev suspensions. In: Proc. Int. Conf. Maglev, CD-ROM. 2004.

[27] Goodall R. Generalised design models for EMS Maglev. In: Proc. Int. Conf. Maglev, CD-ROM. 2008.

[28] Cho D, Kato Y, Spilman D. Sliding model and classical controller in magnetic levitation systems. IEEE Control Syst Mag 1993; 13(1): 42-48.

[29] Sinha PK, Pechev AN. Model reference adaptive control of a maglev system with stable maximum descent criterion. Automatica 1999; 35(8): 1457-1465.

[30] Park JS, Kim JS, Lee JK. Robust control of maglev vehicles with multimagnets using separate control techniques. KSME Int J 2001; 15(9): 1240-1247.

[31] Bittar A, Sales RM. H-2 and H-infinity control for MagLev vehicles. IEEE Control Syst Mag 1998; 18(4): 18-25.

[32] Sinha PK, Pechev AN. Nonlinear H-infinity controllers for electromagnetic suspension systems. IEEE Trans Autom Control 2004; 49(4): 563-568.

[33] Takahashi I, Ide Y. Decoupling control of thrust and attractive force of a LIM using a space vector control inverter. IEEE Trans Ind Appl 1993; 29(1): 161-167. 